\title{
Continuous Landmines Scanning using Legged Robot with Manipulator Arm over Rough Terrain
}

\author{
Dhayaa Khudher, Roger Powell and Maysam Abbod \\ Brunel University London \\ London, UK \\ dhayaa.khudher, roger.powell, maysam.abbod@brunel.ac.uk
}

\begin{abstract}
In this paper, we introduce a new scanning technique for detecting landmines and unexploded ordnance (UXO). The manipulator arm carried by a hexapod robot is capable of scanning rough terrain using a typical mine detector. To speed up the landmine detection and marking, both scanning and moving forward will be done simultaneously. The controller will perform two tasks. The first task is to keep the sensor-head in a fixed level with respect to the ground and the second task is to keep the base of the arm within a specified range of position and orientation. A coupling between the velocity of the end-effector and the velocity of the manipulator base (robot body) is created to ensure the efficiency of the whole process. To verify the feasibility of our design, three experiments will be performed using simulation in MATLAB. The manipulator was modelled using SolidWorks.
\end{abstract}

\section{INTRODUCTION}

As a consequence of decades of conflict, Iraq is a badly affected by landmines and unexploded ordnance (UXO). Hundreds of square kilometres are still contaminated, it is estimated that there are approximately 20 million landmines and millions pieces of UXO [1]. Areas affected include most Iraqi cities, towns, and villages as well as agricultural and other economic land.

In traditional demining methods, the cost of erroneous procedures could include the life of the deminer, even if the mistake was slight. Mines are positioned with increasingly deadly ingenuity and they can be configured as a mixture single and multiple activation devices. Therefore, there is a pressing need to develop fast, safe, effective and efficient demining methods. Nowadays, wheeled, tracked and legged robots are engaged in such duties. Legged robots are preferable over wheeled or tracked robots in demining application as only require a limited number of contact points with the surface, which can be carefully chosen; this ability reduces the probability of triggering landmines [2]. Further, legged robots are inherently omnidirectional, which is a useful characteristic when manoeuvring in small space [3].

In order to delegate a robot to do these tasks, a very efficient controller should be implemented in the robot to give the ability to manoeuvre when environmental uncertainties are present (e.g. uneven terrain). Most robots that are used in demining applications are controlled by the configuration-space methods $[4,5]$. These approaches do not give the ability to use the whole degrees of freedom (DoFs) of the robot efficiently [6]. Development of agile and safe walking robots requires designing the motion in the operation-space rather than configuration-space [7]. The task function approaches $[8,9]$ are proposed to design the motion in a space dedicated to the task to be performed. Then mapping the reference from the task-space to the joint-space is only a numerical problem [10]. These methods are flexible, since the same tasks are generally transposable from one robot to another even in different designs [11]. However, while widely used for fully actuated systems such as conventional manipulators, these methods are not yet standard approaches for legged robots due to their floating base [12] and consequent high number of degrees of freedom.

Moreover, the robots used in demining applications still accomplish the process sequentially [13]. In other words, they interleave the scanning task and task of moving the robot forwards, switching from one to the other. This approach makes the detection operation too slow. To speed up the process both scanning task and moving forward should be fulfilled simultaneously. This complicates the design of the system especially in regard of the floating base of a legged platform since it desirable keep the end-effector moving with as never constant velocity as possible [14]. Therefore, instead of designing the end-effector trajectory as an arc [13], a linear path is suggested. To achieve this, two lines in adjacent boxes are blended by a semi-circular path. This will ensure the continuity of the scanning operation. However, a coupling between the velocity of the robot base (robot body) and the sensor-head velocity should be implemented. More specifically, the nominal velocity of the body will decrease proportionally when the end-effector trajectory encounters an obstacle. Moreover, this coupling will give the capability to accomplish many tasks simultaneously [15].

Instead of finding the control law by analytical solution, an alternative approach is to use quadratic programming (QP). The QP formulation has been used widely in robotics for both inverse kinematics and inverse dynamics. In inverse kinematics, the optimization variable is the robot velocity [16].

The main contributions of this paper are twofold. Firstly, we present a continuous scanning mine detector carried by a legged robot platform. Secondly, we show that coupling the motion of the end-effector velocity and the velocity of the robot's body, in such a way that the sweeping operation is linear, can be done simultaneously.

The rest of the paper is organized as follows: robot platform and virtual environment is summarized in section 2. 
In section 3 the robot mathematical modelling is presented. In section 4 the results of the experiments are discussed. Finally, the paper is concluded in section 5 .

\section{ROBOT PLATFORM AND VIRTUAL ENVIRONMENT}

\section{A. Robot platform}

The robot platform is designed using SolidWorks. Fig. 1 shows a CAD (Computer Aided Design) model of the hexapod robot with six legs placed symmetrically around the body on two sides. The robot has a manipulator arm endowed with a sensor-head for detecting landmines and UXO. The full body of the robot has 24 DoFs $(6 \times 3 \mathrm{DoF})$ for the legs and $(1 \times 6 \mathrm{DoF})$ for the manipulator. The third link of the manipulator has $10 \mathrm{~cm}$ offset from link two to facilitate folding the manipulator over the robot body. To perform various virtual experiments and to obtain required outputs, the CAD model was imported to MATLAB/Sim-Mechanics environment [17]. The specifications and Denavit-Hartenberg transformation of the robot manipulator are listed in table 1.

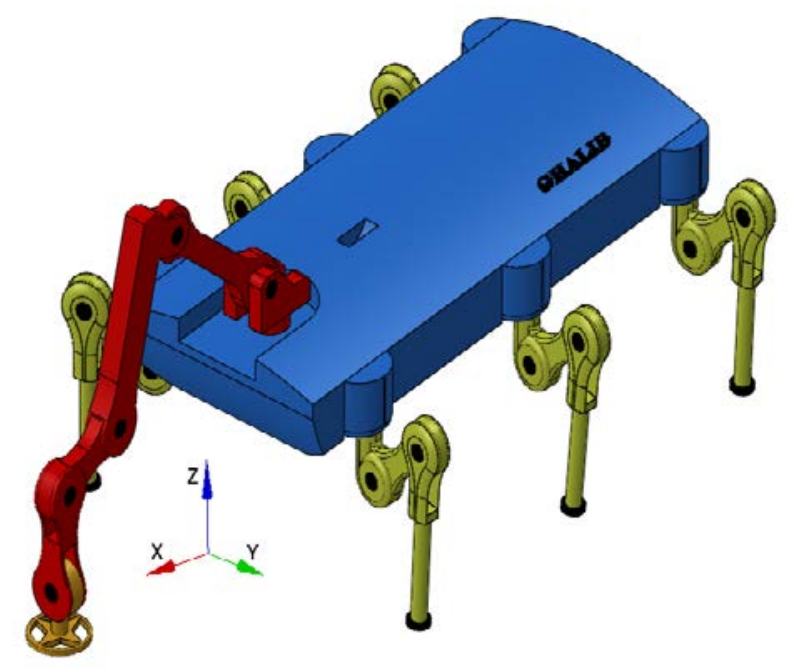

Figure 1. A CAD model of the hexapod robot is designed using SoildWorks 2015.

TABLE I. SPECIFICATION OF THE ROBOT ARM

\begin{tabular}{|c|c|c|c|c|}
\hline$\#$ & $\begin{array}{c}\text { Links length } \\
(\mathrm{cm})\end{array}$ & $\begin{array}{c}\text { Alpha } \\
(\text { deg. })\end{array}$ & Angles & $\begin{array}{c}\text { Joint limit } \\
(\text { deg. })\end{array}$ \\
\hline 1 & 0 & 90 & $\mathrm{q} 1$ & $-90 \sim 90$ \\
\hline 2 & 60 & 0 & $\mathrm{q} 2$ & $-45 \sim 90$ \\
\hline 3 & 60 & 0 & $\mathrm{q} 3$ & $-120 \sim 0$ \\
\hline 4 & 30 & 0 & $\mathrm{q} 4$ & $-90 \sim 90$ \\
\hline 5 & 20 & -90 & $\mathrm{q} 5$ & $-100 \sim 100$ \\
\hline 6 & 20 & 0 & $\mathrm{q} 6$ & $-180 \sim 180$ \\
\hline
\end{tabular}

We assume the minefield that we deal with is divided into boxes each box with $(20 \times 160) \mathrm{cm}$ along $\mathrm{x}$-axis and $\mathrm{y}$-axis respectively. Fig. 2 shows a diagram of a virtual minefield environment. The width of each red box is the same as the reachability of the manipulator arm along y-axis. The distance along $\mathrm{x}$-axis is equal to the diameter of the mine detector.

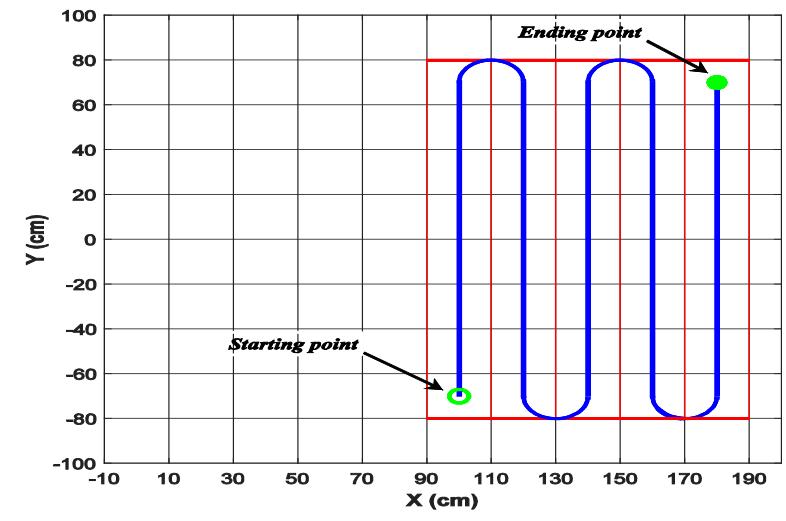

Figure 2. Top view for the minefield with five boxes, the blue line represents the path of the end-effector. The green circle and the solid circle indicate the initial position and final position of the whole path respectively. The boxes of the minefield are illustrated by red lines.

\section{$B$. The utility of the straight-line path}

In this paper, we propose a new strategy for planning the trajectory of the end-effector to travel from side to side within the (red) boxes of the minefield. In this subsection, we argue the advantages of scanning the area in front of the robot in a straight-line. First, it is standard practice to divide the minefield into rectangular segments so a straight-line path will ensure the end-effector passes over the whole area of each box. As a result, the entire area will be scanned homogeneously. Fig. 3 shows a diagram for one box scanned by traditional techniques (an arc trajectory). Further, in contrast with an arc trajectory planning, our trajectory planning will ensure a continuous motion to the sensor-head when travelling through boxes. Having achieved this, the jerk caused by rest-to-rest trajectory will be reduced [18]. Consequently, the effect of the manipulator inertia will be minimized and the overall body balance will be ensured.

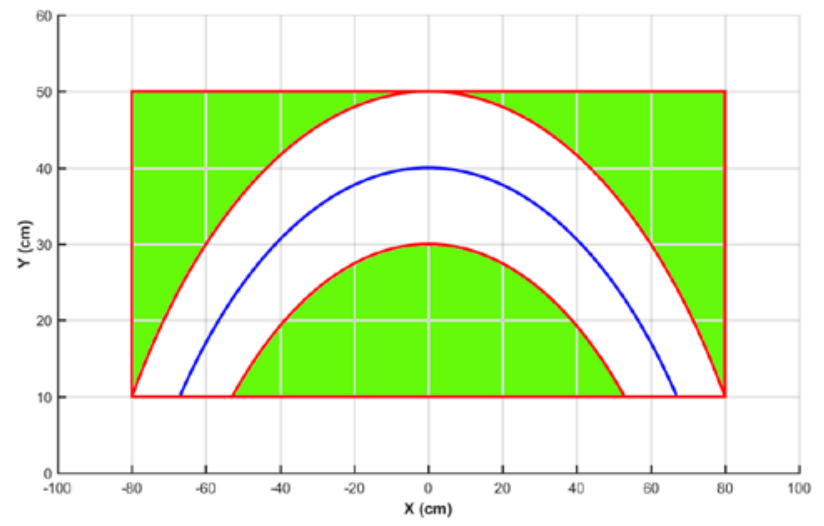

Figure 3. Top view of one box scanned by an arc path planning. The blue line represents the sensor-head path, the white region represents the scanned space of the box and the green zone represents the non-scanned area.

\section{ROBOT MATHEMATICAL MODELLING}

\section{A. Trajectory of the Manipulator End-effector}

The manipulator end-effector is guided to its target with a trajectory created by a fifth order spline. The path is formed from three translation parts along $\mathrm{x}, \mathrm{y}$, and $\mathrm{z}$ axes and three orientation parts (Euler angles). In the first part, the arm tip 
traverses along the y-axis from right and left to scan one box. A semi-circular path is used to blend two linear paths in adjacent boxes. In order to ensure a smooth functioning, each trajectory is assumed to follow a fifth-order polynomial:

$$
q(t)=a_{0}+a_{1} t+a_{2} t^{2}+a_{3} t^{3}+a_{4} t^{4}+a_{5} t^{5}
$$

where $a_{j}, j=1,2,3,4,5$ are the coefficients, whose values are determined using a set of boundary conditions defined over the transition phase for each joint [18].

In case of an obstacle inside a box, the trajectory will be created depending on the height of the obstacle along $\mathrm{z}$-axis and the position of the obstacle in $x-y$ plane. According to this information and the initial position and orientation of the end-effector the new trajectory will be generated. Avoiding an obstacle will be in semi-circular path with a radius depending on its height with respect to the z-axis. In order to determine the height of any obstacle two sensors will be added to the sensor-head and fixed on front of the robot body. A trajectory of the manipulator with an obstacle inside is shown in Fig. 4.

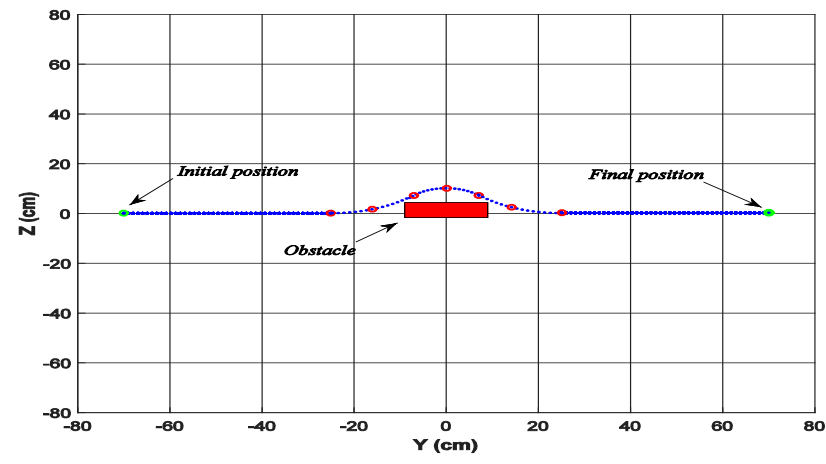

Figure 4. Example of a path of the end-effector with an obstacle inside it. The green circles represent the initial position and final position of the endeffector. The seven red circles represent points depending on the dimensions of the obstacle. The path is interpolated by a linear and parabolic polynomial spline.

\section{B. Quadratic Programming}

The relationship of the end-effector configuration and the angles in the joint-space of the manipulator is represented as follows:

$$
x=f(q)
$$

where $x$ is the position vector of the end-effector and $q$ is the joint angles vector of the arm.

The linear relationship between the velocity in the task-space and the velocity in configuration-space is given by equation (3).

$$
\dot{x}=J \dot{q}
$$

where $\dot{\mathrm{x}}(1 \times 6)$ is the Cartesian velocity vector, $\mathrm{J}(6 \times 6)$ is the Jacobian matrix $\left(\frac{\partial \mathrm{x}}{\partial \mathrm{q}}\right)$ and $\dot{\mathrm{q}}(1 \times 6)$ is the velocity vector of joints.
The inverse kinematics problem includes defining the motion of the robot arm in task-space then mapping the task reference to the configuration according to equation:

$$
\dot{q}=J^{-1} \dot{x}
$$

The limitation of solving equation (4) using the pseudoinverse is the formalization of inequality constraints (obstacle avoidance and joint limit) [19]. An algorithm to calculate the inverse kinematics and to address all constraints, such as equality and inequality constraints, is needed. Therefore, the velocity level is formulated as a Quadratic programming (QP) problem as in the following equations [20].

$$
\begin{gathered}
\min _{\dot{q} \in \Re^{n}} \frac{1}{2} \dot{q}^{T} W \dot{q} \\
\text { s.t.J }(q) \dot{q}-\dot{x}=0 \\
q^{l} \leq q(t) \leq q^{u} \\
\dot{q}^{l} \leq \dot{q}(t) \leq \dot{q}^{u}
\end{gathered}
$$

where $l$ is the lower bound and $u$ is the upper bound for both joint position $q$ and joint velocity $\dot{q}$ respectively, and $\mathrm{W}$ is a weighted matrix. The algorithm solves the linear equation (6) when it has satisfied the inequalities stated in equations (7) and (8).

\section{EXPERIMENTS}

In this section, three experiments will be performed. In the first experiment, the end-effector will scan three boxes. We will consider the problem of avoiding an obstacle with different height and the coupling between the base velocity and the sensor-head velocity. In addition, we will investigate the effect of the variation in the base position and orientation on the end-effector trajectory. We will consider the changes in certain range in the base position along $\mathrm{z}$-axis and a specific twist around $\mathrm{x}$-axis.

\section{A. Arm trajectories with different obstacle heights}

In this experiment, we test the robot motion with different position and height of the mines (or any obstacle). We assume that the landmines lie in different positions along the y-axis. The response of the manipulator to any change in the position of the landmine along the y-axis or to any change in the height with respect to z-axis is shown in Fig. 5. The manipulator scanned three boxes, each box being $20 \mathrm{~cm}$ long and $160 \mathrm{~cm}$ in width. Box one has a mine in the position (80, $0,0)$ with respect to $(\mathrm{x}, \mathrm{y}, \mathrm{z})$ respectively, and with a height 8 $\mathrm{cm}$ over the predefined trajectory. Box two has no mine inside it. The mine in box three is in the position $(120,20,0)$ and its height is $4 \mathrm{~cm}$. It is clear from Fig. 6 that the travelling time of the end-effector to cross through each box is different. The time consumed by the manipulator to scan box one was $16.6 \mathrm{~s}, 8.3 \mathrm{~s}$ for box two, and $16.0 \mathrm{~s}$ for box three. Although there are different situations in each box, the base of the arm (the robot body) traversed same distance. 


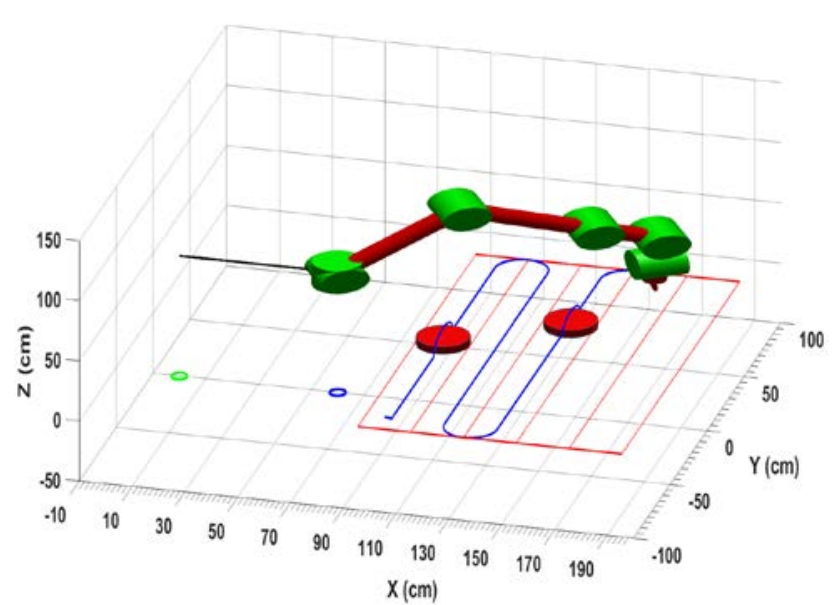

Figure 5. The manipulator arm scanned three boxes. Box one has a mine with a height $8 \mathrm{~cm}$ over the predefined trajectory. The second box has no obstacle inside and the third box has a mine with a height $4 \mathrm{~cm}$ over the predefined trajectory. The green circle represents a projection of the initial base position on the ground. The projection of the final position of the manipulator base is represented by blue circle. The distance between two circles represents the forward motion to the body whilst scanning a sequence of boxes.

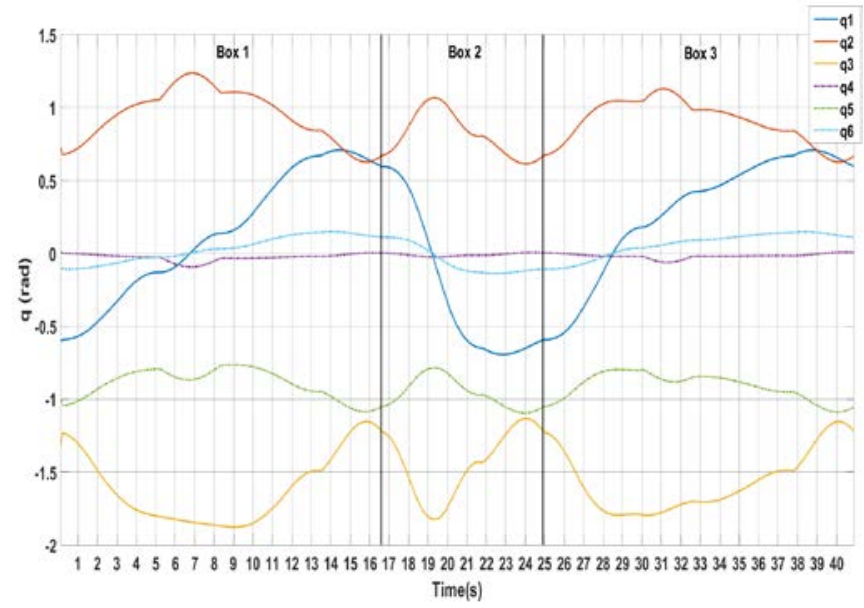

Figure 6. This figure shows three sections separated by two black lines to illustrate the scanning time for each box. The travelling time for box one is $16.6 \mathrm{~s}$. The end-effector needed $8.3 \mathrm{~s}$ to scan box two, and $16.0 \mathrm{~s}$ for last box.

\section{B. Abrupt change in the base position along z-axis}

The following experiment, we attempt to make an abrupt shift to the position of the manipulator base along z-axis by $20 \mathrm{~cm}$ vertically downwards. Fig. 7 illustrates the trajectory of the base (black line) has been changed at time 160s from the position $(20,0,50)$ with respect to xyz axes to the position $(20,0,30)$. As shown in Fig. 8, a small variation in the trajectory occurred. The tiny difference in the path is expectable, because there are four joints $(2,3,4$, and 5$)$ that will recover these changes. Section two of Fig. 8 shows the contribution of joints $(2,3,4$, and 5$)$ to correct the endeffector position after new situation.

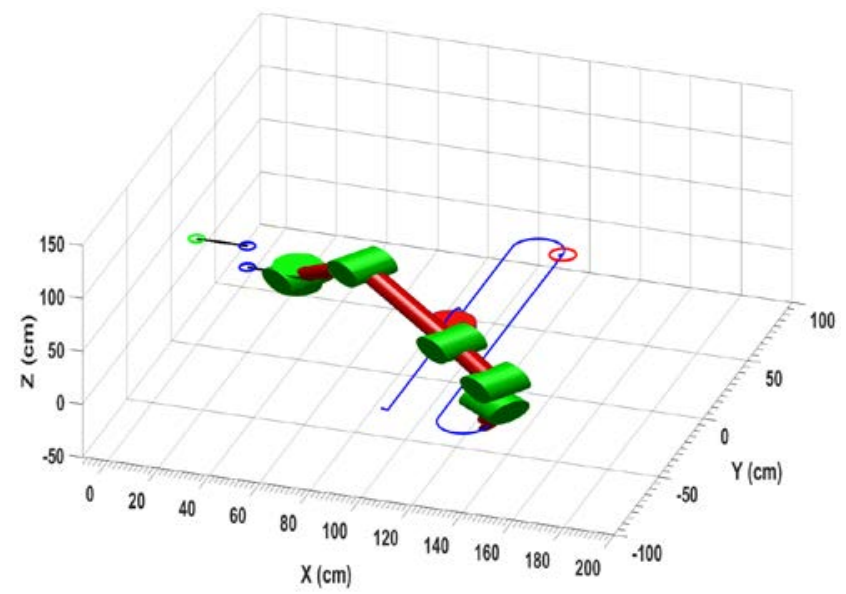

Figure 7. The base position is abruptly changed along z-axis by $20 \mathrm{~cm}$. The green circle represents the initial position of the base; the two blue circles indicate the position before and after changing, and the red circle point on the trajectory at the time of the change.

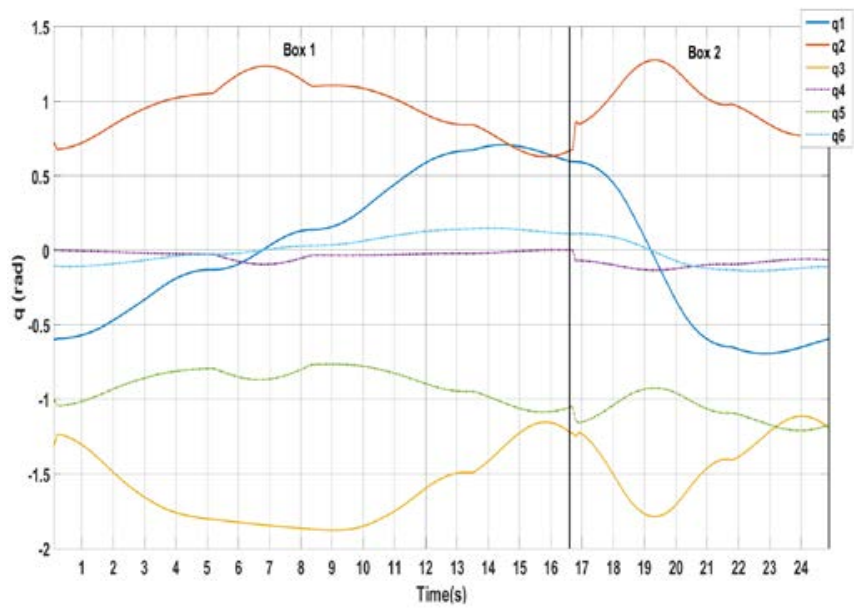

Figure 8. This figure shows two sections separated by black line at time $16.6 \mathrm{~s}$ to illustrate the time consumed to scan box one and box two.

\section{Abrupt change in the base position around $x$-axis}

Next, it is endeavoured to apply a sudden twist on the manipulator base around the $\mathrm{x}$-axis by $\pi / 9$. In order to compare the results and the contribution of the joint angles in this experiment with the previous one, a rotation around $\mathrm{x}$ axis by $\pi / 9$ is applied. Fig. 9 shows the effect of this rotation at time $16.6 \mathrm{~s}$. Although there is a significant deviation in the trajectory, the trajectory has been recovered quickly by the manipulator. Fig. 10 demonstrates the contribution of joint six to correct the end-effector position and orientation. 


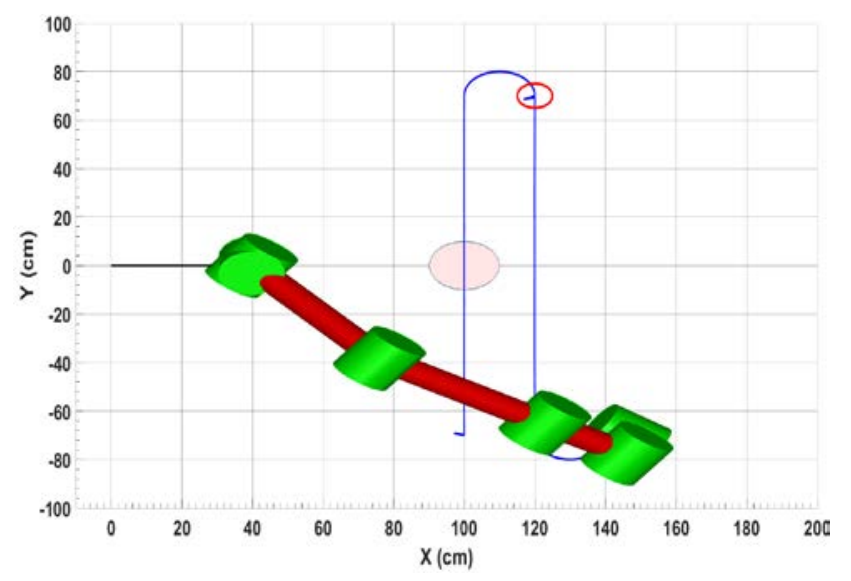

Figure 9. An abrupt change in the base orientation around $\mathrm{x}$-axis by $\pi / 9$. The red circle indicates the effect on the end-effector at the time of the changing in the base orientation around $\mathrm{x}$-axis.

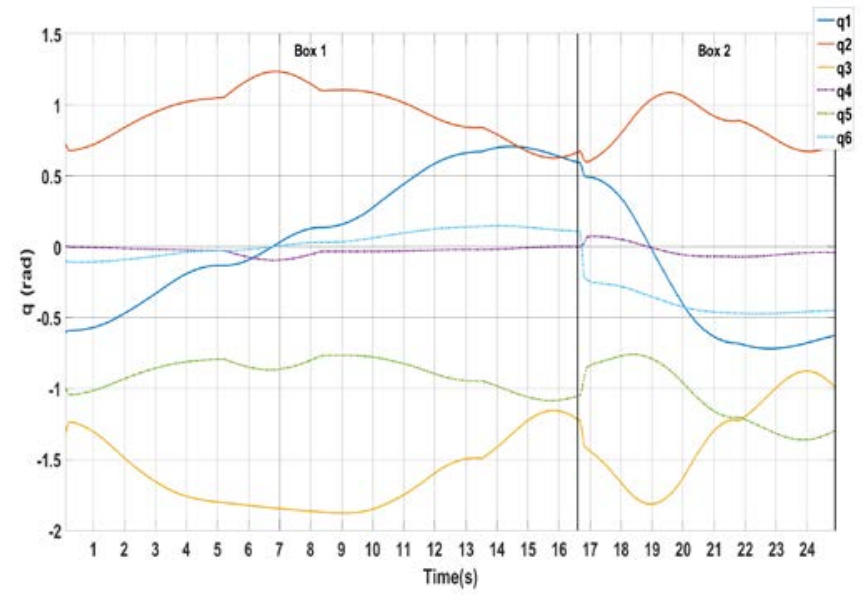

Figure 10. This figure shows two sections separated by black line at time $16.6 \mathrm{~s}$ to illustrate the time consumed to scan box one and box two. The contribution of joint 6 at time $16.6 \mathrm{~s}$ is clear to compensate the variation in the base orientation by $\pi / 9$.

From the above two experiments we conclude that the changing in the base orientation around $\mathrm{x}$-axis has considerable effect on the end-effector trajectory. This is expectable, because the variations in the base position along z-axis will be compensated by four joints (2, 3, 4 and 5). Whereas, the change in the base orientation is recovered by joint 6 .

\section{CONCLUSION AND FUTURE WORK}

In this paper, a continuous landmine-scanning manipulator arm mounted on a six-legged robot is presented. A new trajectory planning has been introduced to scan a minefield. The planning depends on generating a straightline path for the sensor-head to traverse from side to side. The trajectories between adjacent boxes are blended by a semi-circular path. In contrast to the techniques that use a rest-to-rest method, the effect of the jerk caused by the arm is alleviated using our approach. Three experiments verified the efficiency of the controller and the trajectory planner. In the first experiment, the effectiveness of the manipulator to avoid obstacles with different heights and coupling between the velocity of the end-effector and the velocity of the arm base (robot body) was presented. The variation in position and orientation in the base of the manipulator is introduced in the last two experiments. From these experiments, it can be concluded that the prismatic change along z-axis will be compensated by the manipulator (four joints). The variation around $\mathrm{x}$-axis will be recovered by one joint of the manipulator (joint 6). Therefore, the base of the arm should be in a certain range of position and orientation.

In the future work, the motion of the robot body and the motion of the manipulator will be integrated in such a way as to keep the base of the arm in a certain range.

\section{ACKNOWLEDGMENT}

The first author is extremely grateful to the financial support from the Iraqi Ministry of Higher Education and Scientific Research and University of Basra.

\section{REFERENCES}

[1] "The Middle East". U.S. Department of State. N.p., 2017. Web. 27 Feb. 2017.

[2] K. Nonami, Q. Huang, D. Komizo, Y. Fukao, Y. SHIRAISHI, M. FUJIMOTO, and Y. IKEDO, "Development and control of mine detection robot comet-ii and comet-iii,” JSME International Journal Series C Mechanical Systems, Machine Elements and Manufacturing, vol. 46,no.3, pp. 881-890, 2003.

[3] L. Marques, A. De Almeida, M. Armada, R. Fern'andez, H. Montes, P. Gonz'alez, and Y. Baudoin, "State of the art review on mobile robots and manipulators for humanitarian demining," in Proceedings of the $10^{\text {th }}$ International IARP Workshop HUDEM. Citeseer, 2012, p. 1.

[4] T. Evans, R. Ponticelli, E. Garcia, P. Gonzalez de Santos, and M. Armada, "A scanning robotic system for humanitarian de-mining activities,” Industrial Robot: An International Journal, vol. 35, no. 2, pp. 133-142, 2008.

[5] H. Najjaran and A. A. Goldenberg, "Landmine detection using an autonomous terrain-scanning robot," Industrial Robot: An International Journal, vol. 32, no. 3, pp. 240-247, 2005.

[6] L. Sentis and O. Khatib, "A whole-body control framework for humanoids operating in human environments," in Robotics and Automation, 2006. ICRA 2006. Proceedings 2006 IEEE International Conference on. IEEE, 2006, pp. 2641-2648.

[7] M. Hutter, H. Sommer, C. Gehring, M. Hoepflinger, M. Bloesch, and R. Siegwart, "Quadrupedal locomotion using hierarchical operational space control," The International Journal of Robotics Research, vol. 33, no. 8, pp. 1047-1062, 2014.

[8] O. Khatib, "A unified approach for motion and force control of robot manipulators: The operational space formulation," IEEE Journal on Robotics and Automation, vol. 3, no. 1, pp. 43-53, 1987.M. Young, The Technical Writer's Handbook. Mill Valley, CA: University Science, 1989.

[9] Y. Nakamura, H. Hanafusa, and T. Yoshikawa, "Task-priority based redundancy control of robot manipulators,” The International Journal of Robotics Research, vol. 6, no. 2, pp. 3-15, 1987.

[10] A. Del Prete, N. Mansard, F. Nori, G. Metta, and L. Natale, "Partial force control of constrained floating-base robots," in 2014 IEEE/RSJ International Conference on Intelligent Robots and Systems. IEEE, 2014, pp. 3227-3232.

[11] L. Saab, O. E. Ramos, F. Keith, N. Mansard, P. Soueres, and J.-Y. Fourquet, "Dynamic whole-body motion generation under rigid contacts and other unilateral constraints," IEEE Transactions on Robotics, vol. 29, no. 2, pp. 346-362, 2013.

[12] L. Righetti, J. Buchli, M. Mistry, M. Kalakrishnan, and S. Schaal, "Optimal distribution of contact forces with inverse-dynamics 
control," The International Journal of Robotics Research, vol. 32, no. 3, pp. 280-298, 2013.

[13] J. Gavilanes, R. Fern'andez, H. Montes, J. Sarria, P. G. de Santos, and M. Armada, "Instrumented scanning manipulator for landmines detection tasks," in Autonomous Robot Systems and Competitions (ICARSC), 2015 IEEE International Conference on. IEEE, 2015, pp. 180-185.

[14] R. Ponticelli and P. G. De Santos, "Obtaining terrain maps and obstacle contours for terrain-recognition tasks,” Mechatronics, vol. 20, no. 2, pp. 236-250, 2010.

[15] J. Nakanishi, R. Cory, M. Mistry, J. Peters, and S. Schaal, "Operational space control: A theoretical and empirical comparison," The International Journal of Robotics Research, vol. 27, no. 6, pp. 737-757, 2008.

[16] M. Mistry, J. Nakanishi, G. Cheng, and S. Schaal, "Inverse kinematics with floating base and constraints for full body humanoid robot control," in Humanoids 2008-8th IEEE-RAS International Conference on Humanoid Robots. IEEE, 2008, pp. 22-27.

[17] P. Corke, Robotics, vision and control: fundamental algorithms in MATLAB. Springer, 2011, vol. 73.

[18] B. Siciliano, L. Sciavicco, L. Villani, and G. Oriolo, Robotics: modelling, planning and control. Springer Science \& Business Media, 2010.

[19] O. Kanoun, F. Lamiraux, and P.-B. Wieber, "Kinematic control of redundant manipulators: Generalizing the task-priority framework to inequality task," IEEE Transactions on Robotics, vol. 27, no. 4, pp. 785-792, 2011.

[20] A. Escande, N. Mansard, and P.-B. Wieber, "Hierarchical quadratic programming: Fast online humanoid-robot motion generation,” The International Journal of Robotics Research, vol. 33, no. 7, pp. 10061028, 2014. 